\title{
Proposed Optical Random Access CDMA Protocol with Stop \& Wait ARQ
}

Yousef M. Abd-El Malek, Student Member, IEEE, Ziad A. El-Sahn, Student Member, IEEE Hossam M. H. Shalaby, Senior Member, IEEE, El-Sayed A. El-Badawy, Senior Member, IEEE

Department of Electrical and Computer Engineering, Faculty of Engineering, Alexandria University, Alexandria 21544, Egypt.

Abstract - A new random access protocol for optical code division multiple access (CDMA) communication systems is proposed. Stop \& wait automatic repeat request (ARQ) is implemented at the optical link layer. A detailed state diagram and a mathematical model based on the equilibrium point analysis (EPA) technique are presented. Several performance measures are evaluated under different network parameters. In our numerical calculations both chip-level and correlation models have been adopted at the receiver side. Furthermore, the effect of thermal noise is studied. We also compare the performance of the proposed protocol to that of the round robin receiver/transmitter $\left(R^{3} T\right)$ protocol which is based on a go back-n technique. We prove by numerical analysis that the proposed protocol is less complex and significantly outperforms the $R^{3} T$ protocol. Our results also reveal that the performance of the proposed protocol with correlation receivers is competitive to that of the $R^{3} T$ protocol with chip-level receivers, which reduces the overall system cost.

Keywords - Chip-level receivers, code-division multiple-access, correlation receivers, on-off keying, optical CDMA protocols, optical link layer, optical networks, thermal noise.

\section{I- INTRODUCTION}

The success of modern communication systems has shifted the focus towards optical fiber networks. Especially, optical code division multiple access (CDMA) systems have been shown to be competitive candidates to support a large number of simultaneous users [1]-[13]. Most of researches in optical CDMA networks were concentrated in the physical layer. There are, however, a few authors that have examined the network or link layer of optical CDMA communication systems, [6]-[10]. In [6], [7] Hsu and Li 
have studied the slotted and unslotted optical CDMA networks. In [8] Shalaby has proposed two media access control (MAC) protocols for optical CDMA networks. However the effect of multi-packet messages, connection establishment and corrupted packets haven't been taken into account. Recently, Shalaby [9] has developed a new protocol called round robin receiver/transmitter $\left(R^{3} T\right)$ protocol that has solved some of the above problems. The $R^{3} T$ protocol is based on a go-back $n$ automatic repeat request (ARQ), that is when a packet gets corrupted, the transmitter retransmits it and all subsequent packets. This scenario gives good performance for low population networks, while the performance is still low for larger population networks. Considering only the retransmission of corrupted packets, a selective reject ARQ has been applied in [10], which yields better results in case of higher population networks.

Our goal in this paper is to develop a new optical random access CDMA protocol which is based on a stop \& wait ARQ in order to reduce the complexity of the previously proposed protocols. At the same time we aim at improving the system performance compared to the $R^{3} T$ protocol. Moreover, the proposed protocol is examined for the case of both chip-level and correlation receivers. In most cases of practical interest, thermal noise dominates the receiver performance. Accordingly, in our analysis we will study the effect of thermal noise and its impact on the performance of the proposed protocol. The rest of this paper is organized as follows. In Section II we discuss the system and hardware architecture for our optical CDMA network. Section III is devoted for a description of the proposed protocol. The optical link layer is investigated and a complete state diagram of this protocol is presented. Section IV is maintained for the mathematical model, where derivations of the steady state system throughput, the average packet delay, the protocol efficiency, and the blocking probability are given. In Section V we discuss some of the numerical results obtained. A comparison between the proposed protocol and the $R^{3} T$ protocol, [9] is also considered. Finally we give our conclusions in Section VI.

\section{II- SYSYTEM \& HARDWARE ARCHITECTURE}

Considering the network topology at the physical layer we have a passive optical star network composed of a star coupler connecting $N$ users as shown in Fig. 1. Optical orthogonal codes (OOCs) are used because they have both a peak cross-correlation and 
a shifted autocorrelation equal to one. The choice of code weight $w$ and code length $L$ for OOCs is arbitrary but these quantities determine the cardinality according to [1]:

$$
|C|=\left\lfloor\frac{L-1}{w(w-1)}\right\rfloor,
$$

where $\lfloor x\rfloor$ denotes the integer portion of the real value $x$. Due to the bursty nature of the traffic, we allow the number of users to exceed the number of available codes $|C|$.

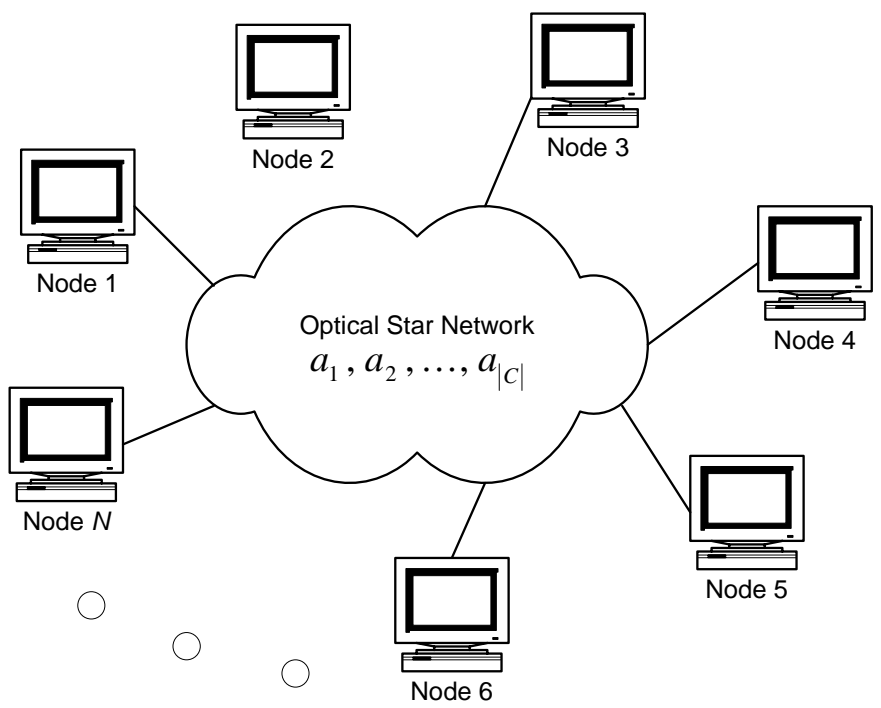

Fig. 1. Optical CDMA Network Topology.

In our model, we assume that all codes are always available in a pool, Fig. 1. In order to avoid receiver tunability, codes are assigned to users apriori. That is, when a user subscribes to the network, it is given a code (possibly used) randomly. Further, a code is randomly cyclic shifted around itself once assigned. In this way there is no need for pretransmission coordination. It has been shown that for fixed data rate and chip duration, there is no advantage in using pulse position modulation (PPM) in place of onoff keying (OOK). That's why each user is able to generate an optical OOK-CDMA signal according to its signature code that represents its data.

Chip-level receivers are implemented at the physical layer of our optical CDMA network because of their ability to overcome the effect of multiple access interference (MAI), [4]. Assuming that there are $r \in\{1,2, \cdots, N\}$ active users, we define $k \in\{0,1,2, \cdots r-1\}$, and $m \in\{0,1, \cdots r-1-k\}$ as the number of users that interfere with the desired user at exactly 1 chip and $w$ chips, respectively. In addition let $k_{i}$ denote 
the number of users that interfere with the desired user at weighted chip $i$. Thus we have $k=\sum_{i=1}^{w} k_{i}$. Considering a message that is composed of $\ell>0$ packets, each having $K>0$ bits and taking only the effect of MAI into account, we can write the packet success probability as follows [8]:

$$
\begin{aligned}
P_{S}(r)= & \sum_{k=0}^{r-1} \sum_{m=0}^{r-1-k} \frac{(r-1) !}{k ! m !(r-1-m-k) !} \cdot p_{1}^{k} p_{w}^{m}\left(1-p_{1}-p_{w}\right)^{r-1-m-k} \\
& \cdot \sum_{\substack{k_{1}, k_{2}, \cdots, k_{w} \\
k_{1}+\cdots+k_{w}=k}} \frac{k !}{k_{1} ! \cdots k_{w} !} \cdot\left(\frac{1}{w}\right)^{k} \\
& \left.\cdot \frac{1}{2}+\frac{1}{2^{m+1}}\left(\sum_{i=1}^{w} \frac{1}{2^{k_{i}}}-\sum_{i=1}^{w-1} \sum_{j=i+1}^{w} \frac{1}{2^{k_{i}+k_{j}}}+\cdots+(-1)^{w-1} \frac{1}{2^{k}}\right)\right]^{K}
\end{aligned}
$$

Here, $p_{1}$ and $p_{w}$ denote the probability of 1 and $w$ chip-interferences, respectively between two users. They are given by [8]

$$
p_{w}=\frac{1}{L} \cdot\left\lfloor\frac{L-1}{w(w-1)}\right\rfloor^{-1}, \quad p_{1}=\frac{w^{2}}{L}-w p_{w} .
$$

The packet success probability including the effect of the receiver's thermal noise can be given by [12]:

$$
\begin{aligned}
P_{S}(r) & =\sum_{k=0}^{r-1} \sum_{m=0}^{r-1-k} \frac{(r-1) !}{k ! m !(r-1-m-k) !} \cdot p_{1}^{k} p_{w}^{m}\left(1-p_{1}-p_{w}\right)^{r-1-m-k} \cdot \sum_{\substack{k_{1}, k_{2}, \cdots, k_{w}: k \\
k_{1}+\cdots+k_{w}=k}} \frac{k !}{k_{1} ! \cdots k_{w} !} \cdot\left(\frac{1}{w}\right)^{k} \\
\cdot & {\left[\frac{1}{2}-\frac{1}{2} \sum_{b=0}^{1}(-1)^{b} \sum_{i=1}^{w}(-1)^{i}\left(\begin{array}{c}
i \\
w
\end{array}\right) \sum_{u=o}^{m}\left(\begin{array}{l}
m \\
u
\end{array}\right)\left(\frac{1}{2}\right)^{m} \cdot \prod_{j=1}^{i} \sum_{v_{j}=0}^{k_{j}}\left(\begin{array}{l}
k_{j} \\
v_{j}
\end{array}\right)\left(\frac{1}{2}\right)^{k_{j}} Q\left(\frac{m_{b j}-\theta}{\sigma_{b j}}\right)^{K},\right.}
\end{aligned}
$$

where $b \in\{0,1\}$ is the user data bit, $\theta$ is the decision threshold, and $m_{b j}$ and $\sigma_{b j}^{2}$ are the conditional mean and variance of the decision variable (photon count per marked chip position), respectively, and can be found in [12].

The packet success probability of the correlation receiver without hard-limiters can be computed as in [8], where the effect of the receiver's shot and thermal noises have been neglected. 


\section{III- MATHEMATICAL MODEL}

Because of its simplicity over other ARQs, stop \& wait is implemented at the data link layer of our optical CDMA network. After a packet is sent, a user enters a waiting mode to get a feedback of that packet. If a positive acknowledgement is received the user will send the next packet, otherwise he will retransmit another version of the corrupted packet. We impose the following assumptions in our model for optical CDMA protocol:

- Time is slotted with slot size $T_{s}$, a two way propagation delay time is assumed to be equal to $t$ time slots, and a timeout duration of $\tau$ time slots is selected such that $1 \leq \tau \leq t$

- A message is composed of $\ell>t$ packets each having $K>0$ bits. One packet should fit in a time slot $T_{s}=K \ell T_{c}$, where $T_{c}$ is the chip duration.

- A maximum of 1 message can arrive at each time slot to a station with probability A (also called user activity). This message is stored in a buffer till its successful transmission.

- Any arrival to a non empty buffer will be blocked.

- Connection requests and acknowledgements are exchanged between stations.

- Transmission times for requests and acknowledgements are neglected.

- A priority is given for the reception mode than for the transmission mode.

- Receivers use a cyclic redundancy check (CRC) to determine whether a received packet is correctly detected or not.

The complete state diagram of the proposed optical CDMA random access protocol with stop \& wait ARQ is illustrated in Fig. 2. Each state is labeled by its number of users. Transition between states is on a slot basis; that is the duration of each state equals to one time slot. At any time slot, any user in the network will be in one of the following states or modes:

- Initial state, $\{m\}$. Users in the initial state scan across all codes in a round-robin manner. If a connection request (event happening with probability $\sigma$ ) is found, a station will proceed to the acknowledgement mode. If there is a message arrival and there is no connection request, it will go to the requesting mode. If there is neither message arrival nor connection request, the station will remain in the initial state.

- Requesting mode, $\left\{q_{1}, q_{2}, \ldots, q_{\tau}\right\}$. Stations in this mode send repeated requests $\left\{q_{1}, q_{2}, \ldots, q_{\tau}\right\}$ for $\tau$ time slots. Then the station should wait for a feed back and 
thus enters a waiting mode $\left\{W_{1}^{q}, W_{2}^{q}, \ldots, W_{t-1}^{q}\right\}$, for $t-1$ time slots, as depicted in Fig. 2. Whenever a waiting station gets a positive acknowledgement (event occurring with probability $\gamma$ ) from the destination, it starts sending its message and enters the transmission mode, otherwise it remains in the waiting mode. In the last waiting state, if an acknowledgement is not received, the station is timed out and returns to the initial state.

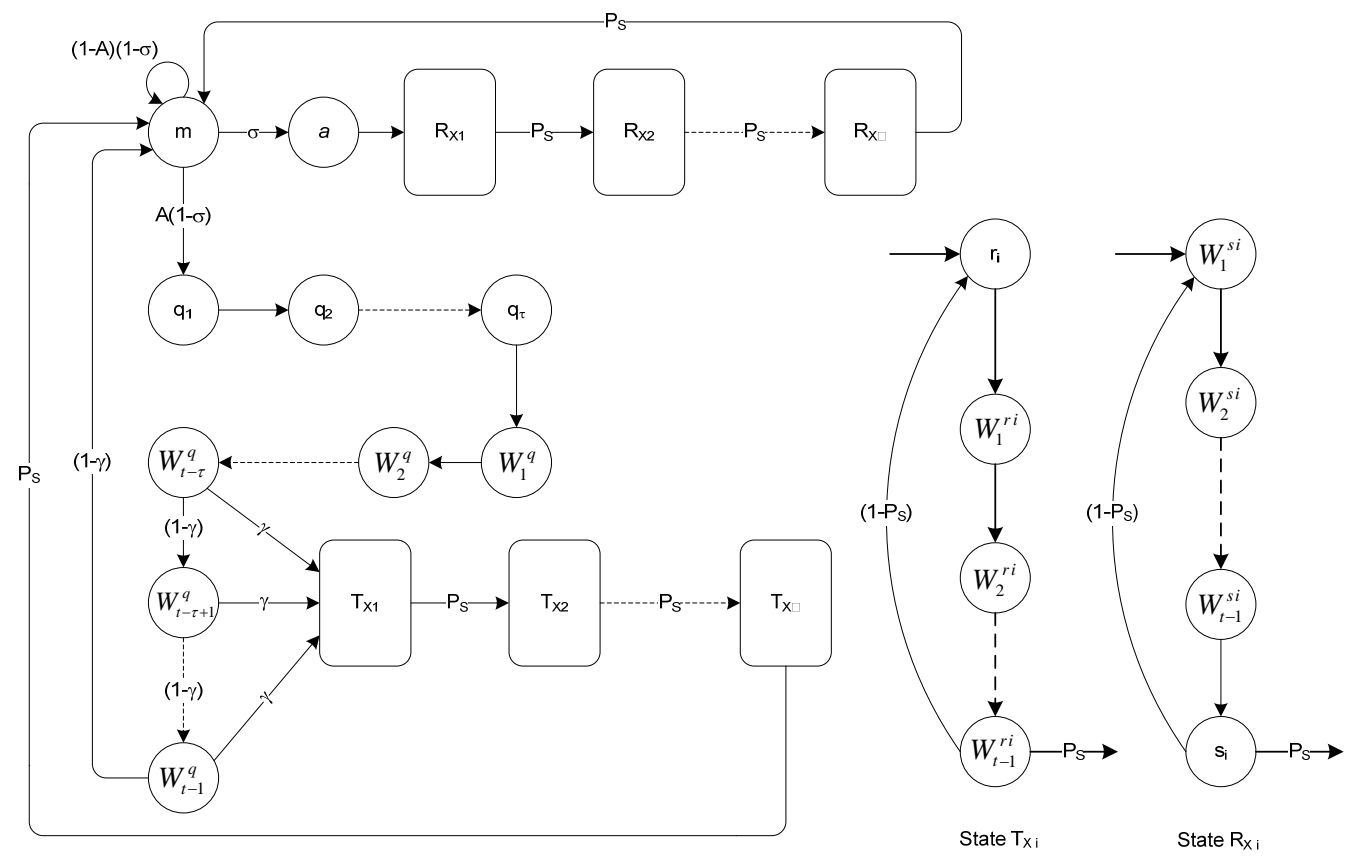

Fig. 2. Complete state diagram of the proposed Stop \& Wait optical CDMA protocol.

- Acknowledgement state, $\{a\}$. In this state, the station sends an acknowledgement to a requesting station and then enters a waiting mode $\left\{W_{1}^{s 1}, W_{2}^{s 1}, \ldots, W_{t-1}^{s 1}\right\}$ for $t$ time slots till the reception of the first packet.

- Reception mode, $\left\{R x_{1}, R x_{2}, \ldots, R x_{\ell}\right\}$. A user in the reception mode receives either new packets or retransmitted ones. Fig. 2 illustrates the structure of the states $R_{X i}$, $i \in\{1,2, \ldots, \ell\}$. In states $s_{i}$, a user receives packet $i$, if it is successfully received, the user will move to states $\left\{W_{1}^{s i+1}, W_{2}^{s i+1}, \ldots, W_{t-1}^{s i+1}\right\}$ waiting for the next packet, otherwise he will ask for retransmission and enters a waiting mode 
$\left\{W_{1}^{s i}, W_{2}^{s i}, \ldots, W_{t-1}^{s i}\right\}$. If the station receives the last packet successfully it goes back to the initial state.

- Transmission mode, $\left\{T x_{1}, T x_{2}, \ldots, T x_{\ell}\right\}$. This mode involves transmission states $r_{i}$, $i \in\{1,2, \ldots, \ell\}$ and waiting states $W_{j}^{r i}, j \in\{1,2, \ldots, t-1\}$, Fig. 2. A user in state $r_{i}$ is transmitting packet $i$, then he enters a waiting mode $\left\{W_{1}^{r i}, W_{2}^{r i}, \ldots, W_{t-1}^{r i}\right\}$ to get the acknowledgement of that packet. If a positive feedback is received the user will proceed to state $r_{i+1}$ otherwise he will return to state $r_{i}$ for retransmission. After successful transmission of the last packet the user will return to the initial state.

\section{IV- PERFORMANCE ANALYSIS}

Because of the complexity of the above model and the prohibitively large number of states, the problem will be analytically intractable if we try to calculate the transition probabilities between states and the stationary probabilities using Markov chains [13]. Fortunately, the equilibrium point analysis (EPA) technique significantly simplifies the problem. In this technique, the system is always assumed to be operating at an equilibrium point [11]; that is the number of users entering a state is equal to the number of users departing from the same state. By writing down the flow equations for each state, the performance of the proposed optical CDMA protocol can be evaluated.

\section{A. Transmission Mode}

This mode involves states $r_{i}$ and states $W_{j}^{r i}$, where $i \in\{1,2, \ldots, \ell\}$ and $j \in\{1,2, \ldots, t-1\}$. From Fig. 2, we have the following flow equations.

$$
r_{1}=r_{2}=\ldots=r_{\ell} \quad \text { and } \quad W_{j}^{r i}=r_{1} .
$$

Let $r_{o}$ denotes the number of transmitting users in a given slot such that

$$
r_{o}=\sum_{i=1}^{\ell} r_{i}=\ell r_{1}
$$

We define $W^{r}$ as the number of users waiting after transmission

$$
W^{r}=\sum_{i=1}^{\ell} \sum_{j=1}^{t-1} W_{j}^{r i}=\ell(t-1) \cdot r_{1} .
$$




\section{B. Reception Mode}

Assuming that the number of users transmitting packet $i$ is equal to that receiving the same packet, for $i=1$ we can directly write $r_{1}=s_{1}$. From Fig. 2 , we can write the flow equations for states $s_{i}$ and $W_{j}^{s i}$, for $i \in\{1,2, \ldots, \ell\}$ and $j \in\{1,2, \ldots, t-1\}$ as follows:

$$
\begin{aligned}
& W_{1}^{s 1}=a+\left(1-P_{S}\right) \cdot s_{1}, \\
& s_{1}=s_{2}=\ldots=s_{\ell}=r_{1} \quad \text { and } \quad W_{j}^{s i}=r_{1} .
\end{aligned}
$$

We define the following variables

$$
\begin{aligned}
& s=\sum_{i=1}^{\ell} s_{i}=\ell r_{1} \\
& W^{s}=\sum_{i=1}^{\ell} \sum_{j=1}^{t-1} W_{j}^{s i}=\ell(t-1) \cdot r_{1}
\end{aligned}
$$

\section{Acknowledgement Mode}

Again by writing the flow equations for the states in this mode, described in Fig. 1 and using equation (6) we get:

$$
a=\sigma m=P_{S} r_{1} .
$$

Therefore

$$
m=\frac{P_{S}}{\sigma} r_{1}
$$

\section{Requesting Mode}

Fig. 2 illustrates the requesting states and waiting states in the requesting mode. By writing down the flow equations as in [9] we define:

$$
q+W^{q}=\sum_{i=1}^{\tau} q_{i}+\sum_{i=1}^{t-1} W_{i}^{q}=\left[t-1+\frac{1}{\gamma}\left(1-(1-\gamma)^{\tau}\right)\right] A \frac{1-\sigma}{\sigma} P_{S} r_{1} .
$$

The probability that a request is found by a scanning user $\sigma$ and the probability that a station gets an acknowledgement $\gamma$ can be computed as follows [9]:

$$
\begin{aligned}
& \sigma=\frac{1}{2}\left[\sqrt{\left(A P_{S} \tau \frac{r_{1}}{N}\right)^{2}+4\left(A P_{S} \tau \frac{r_{1}}{N}\right)}-A P_{S} \tau \frac{r_{1}}{N}\right] . \\
& \gamma=1-\left[1-\frac{\sigma}{A(1-\sigma)}\right]^{1 / \tau}
\end{aligned}
$$




\section{E. Steady State System Throughput}

The steady state system throughput $\beta(N, A, t, \tau, \ell)$ is defined as the average number of successful received packets per slot. It can be calculated as follows:

$$
\beta(N, A, t, \tau, \ell)=\sum_{i=1}^{\ell} s_{i} \cdot P_{S}=P_{S}\left(r_{o}\right) \ell r_{1} .
$$

Substituting with equation (4) we get

$$
\beta(N, A, t, \tau, \ell)=r_{o} P_{S}\left(r_{o}\right) .
$$

To compute $r_{o}$ we assume that the total number of users in all states is equal to $N$, yielding

$$
\begin{aligned}
& N=m+r+W^{r}+s+W^{s}+a+q+W^{q} \\
& =\frac{r_{o}}{\ell}\left[\frac{P_{S}}{\sigma}+2 \ell t+P_{S}+\left\{t-1+\frac{1}{\gamma}\left(1-(1-\gamma)^{\tau}\right)\right\} A \frac{1-\sigma}{\sigma} P_{S}\right],
\end{aligned}
$$

where we have used equations (4) - (10).

\section{F. Blocking Probability}

The blocking probability is defined as the probability of an arrival being blocked. In this case the blocking probability is equal to the probability that the station is not in the initial state $m$ and there is a message arrival $A$ or the station is in the initial state $m$ but there is a request for connection and at the same time there is a message arrival $A$. Thus, we can write

$$
P_{B}=\frac{m}{N} \cdot \sigma \cdot A+\left(1-\frac{m}{N}\right) \cdot A .
$$

Substituting with equations (4) and (9) we get

$$
P_{B}=A\left[1-\frac{P_{S}}{\ell N}\left(\frac{1-\sigma}{\sigma}\right) r_{o}\right] \text {. }
$$

\section{G. Protocol Efficiency and Average Delay}

The protocol efficiency $\eta$ is defined as the ratio between the number of successfully received packets and the number of packets available for transmission:

$$
\eta=\frac{\beta(N, A, t, \tau, \ell)}{r_{o}} .
$$

The average packet delay $D$ can be calculated from Little's theorem: 


$$
D=\frac{N A \cdot\left(1-P_{B}\right)}{\beta(N, A, t, \tau, \ell)} \quad \text { slots, }
$$

where $N A \cdot\left(1-P_{B}\right)$ denotes the total traffic in the network.

Note that equations (13) - (15) are valid for both optical random access CDMA protocols; with Stop \& Wait ARQ and with go-back $n$ ARQ [9].

\section{V- NUMERICAL RESULTS}

In this section, we discuss some numerical results for the proposed optical random access CDMA protocol. The steady state system throughput, the blocking probability, the average packet delay, and the protocol efficiency derived above have been evaluated and compared to the results in [9]. The performance of the proposed protocol using both chip-level receivers and correlation receivers is also presented. Correlation receivers are only considered in Fig. 3. Finally, the effect of thermal noise is taken into consideration in the last figure.

Table 1. Parameters used for numerical calculations.

\begin{tabular}{lll}
\hline CDMA Parameters & \multicolumn{2}{c}{ APD Parameters (thermal noise) } \\
\hline$R_{b}=127 \mathrm{Mbps}$ & APD responsivity at unity gain & $R=0.84 \mathrm{~A} / \mathrm{W}$ \\
$L=31$ & APD dark current & $I_{d}=1 \mathrm{nA}$ \\
$w=3$ & Average APD gain & $G=100$ \\
$K=127$ & APD effective ionization ratio & $k_{\text {eff }}=0.02$ \\
& Receiver noise temperature & $T^{o}=300 \mathrm{~K}$ \\
& Receiver load resistor & $R_{L}=50 \Omega$ \\
\hline
\end{tabular}

Our results are plotted in Figs. 3-8. A two way propagation delay time $t \in\{2,4,6\}$ slots (or interstation distances of $z=v T_{s} t / 2 \in\{200,400,600\} \mathrm{m}$, where $v \approx 2 \times 10^{8} \mathrm{~m} / \mathrm{s}$ is the speed of light inside a fiber) and a timeout duration $\tau=1$ slot are imposed in our simulations. Table 1 shows the remaining parameters used for the numerical calculations. 


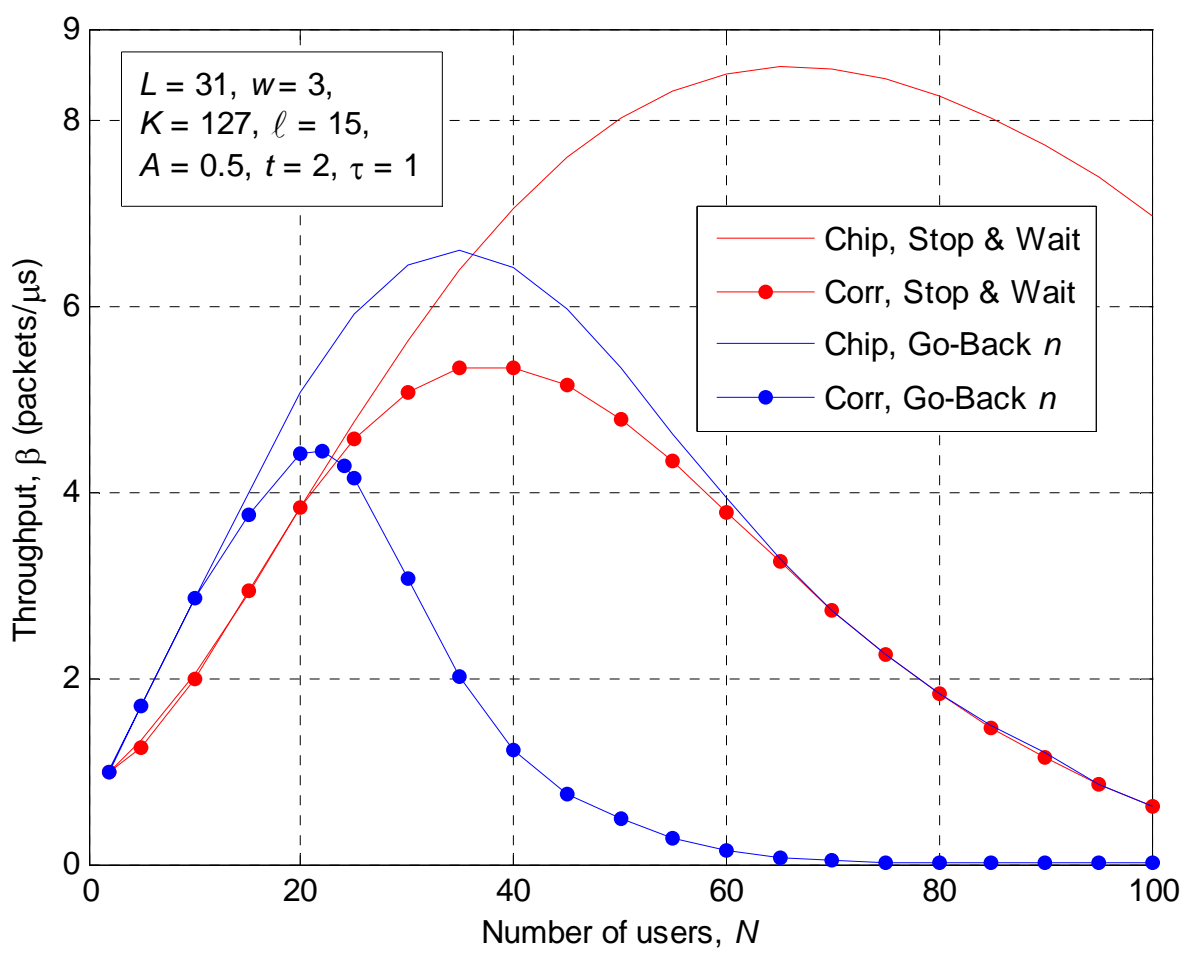

Fig. 3. Throughput vs. number of users for different receivers.

In Fig. 3 we have plotted the throughput versus the number of users for both chip-level receivers and correlation receivers. General trends of the curves can be noticed. As the number of users in the network increases more packets become available for transmission with low interference. Thus the throughput increases till it reaches its peak. As the number of users is further increased the effect of MAI becomes significant and the throughput starts to decay. In the case of stop \& wait ARQ the protocol supports a larger number of users and reaches higher values of throughput. This is because the channel is not busy all the time, as users enter a waiting mode after sending each packet. On the other hand for a protocol depending on a go-back $n$ ARQ, users continuously send their packets, which contributes to higher traffic loads, yielding lower packet success probabilities and lower throughput values. It can be inferred that the performance of both protocols is reduced when using correlation receivers and that the performance of the stop \& wait with correlation receivers is nearly close to that of the $R^{3} T$ protocol with chip-level receivers. 


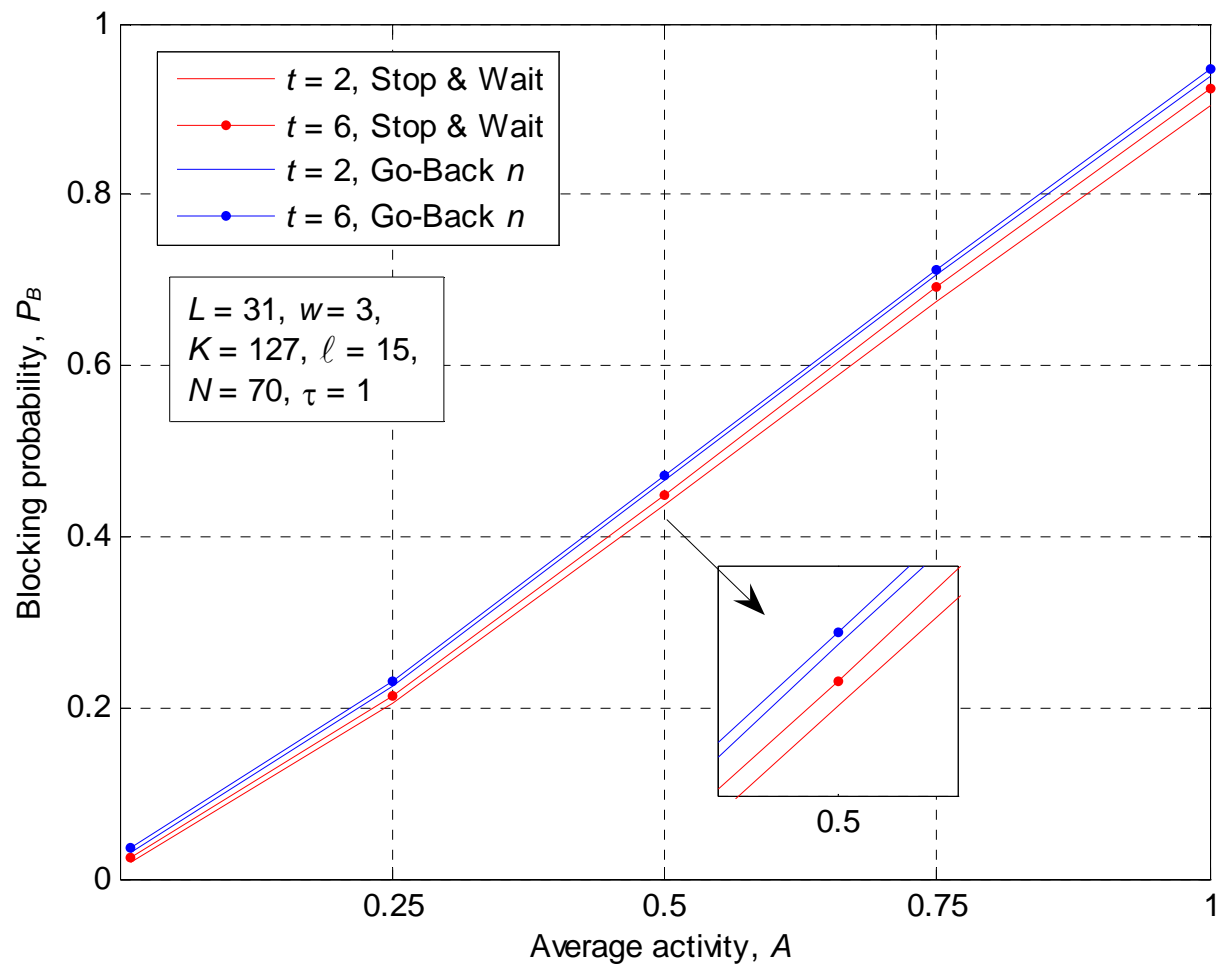

Fig. 4. Blocking probability vs. user activity for different interstation distances.

In Fig. 4 the blocking probability has been plotted versus user activity for different activity levels and for different interstation distances. It can be seen that the blocking probability increases significantly for higher activity levels, $A \in\{0.75,1\}$. The proposed protocol (stop \& wait) provides lower blocking probabilities because at $N=70$ this protocol can still handle the traffic as depicted in Fig. 3. For larger intersatation distances, the channel will be busy for longer durations. This gives rise to a high probability of collision between packets which reduces the throughput, therefore the blocking probability increases.

In Fig. 5 we have plotted the system throughput and the average packet delay versus the average activity for different number of users $N \in\{30,70\}$. It can be noticed that the $R^{3} T$ protocol exhibits higher throughput values and lower delays at $N=30$. Whereas the proposed protocol outperforms the $R^{3} T$ protocol at $N=70$, as argued in Fig. 3. For short interstation distances, as the user activity increases the throughput also increases till it reaches saturation whereas for longer distances the throughput falls after reaching its peak. In fact the initial increase of throughput is because as $A$ increases above zero, 
more packets become available with low interference. The throughput decay in the case of long propagation delays after reaching its peak because the number of active users increases while other users already in the transmission mode are still busy transmitting their messages over long distances. The interference would thus increase rapidly and packet failures become more probable. Finally, it is noticed that for longer interstation distances and an increase in user activity, the average packet delay increases.
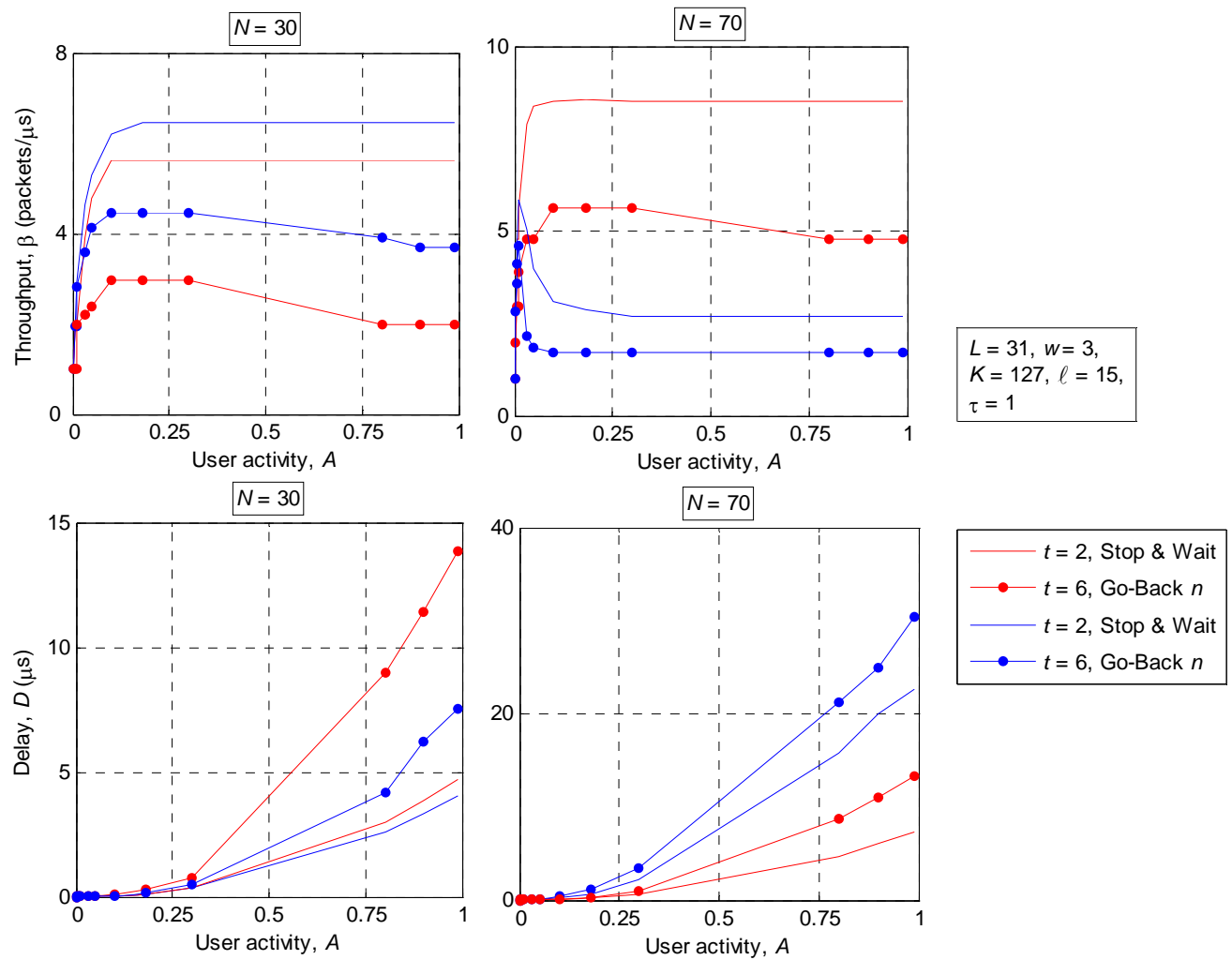

Fig. 5. Throughput and delay vs. activity for different number of users and different interstation distances.

The relation between the system throughput and the number of users for our proposed protocol is depicted in Fig. 6. It can be seen that for longer propagation delays, the proposed protocol accommodates a higher number of users. Also the quality of service (QOS) requirements is achieved in a large dynamic range. This is because as $t$ increases, users wait longer times after transmitting their packets, giving chance to other users to start transmission. 


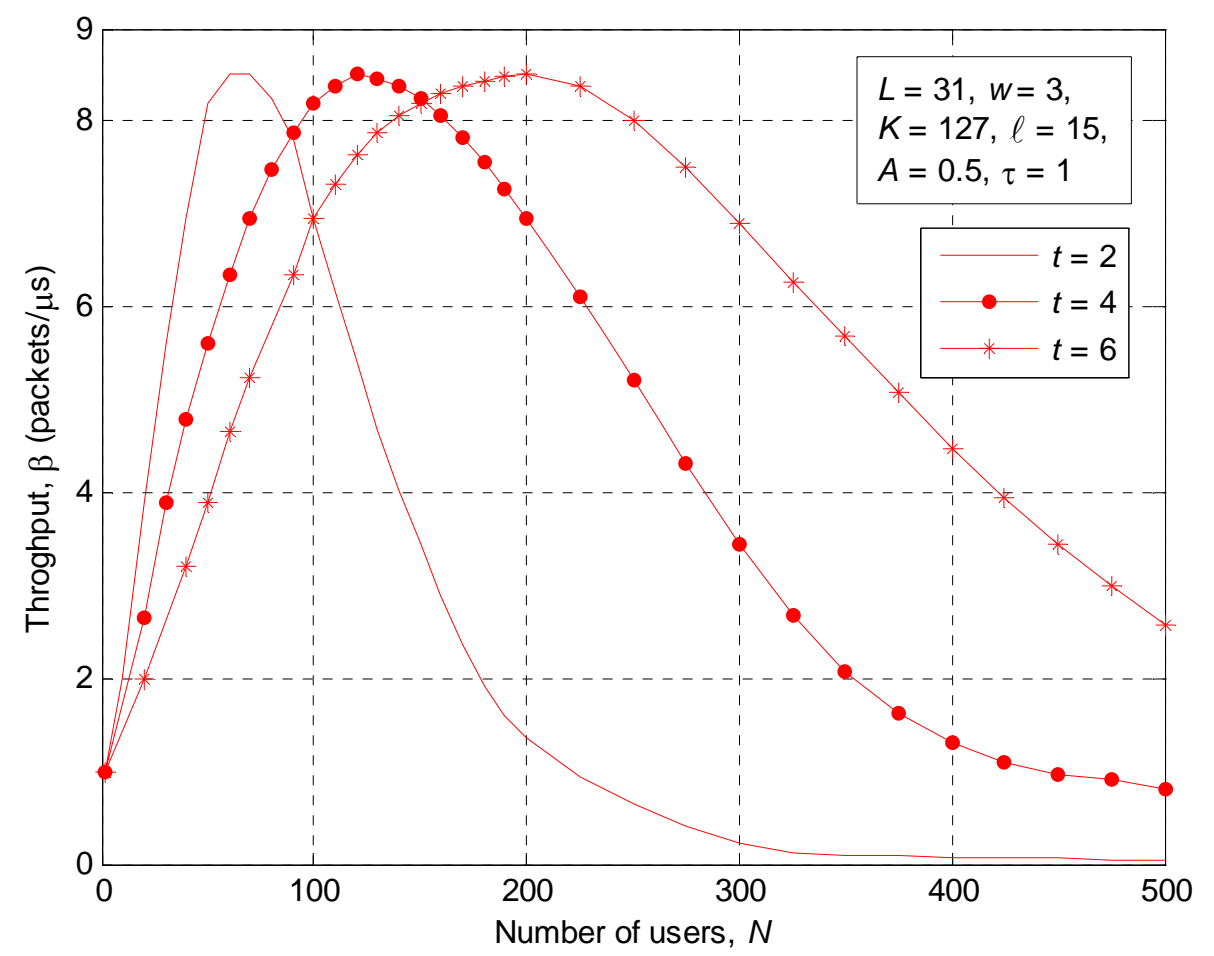

Fig. 6. Throughput vs. number of users for different interstation distances.

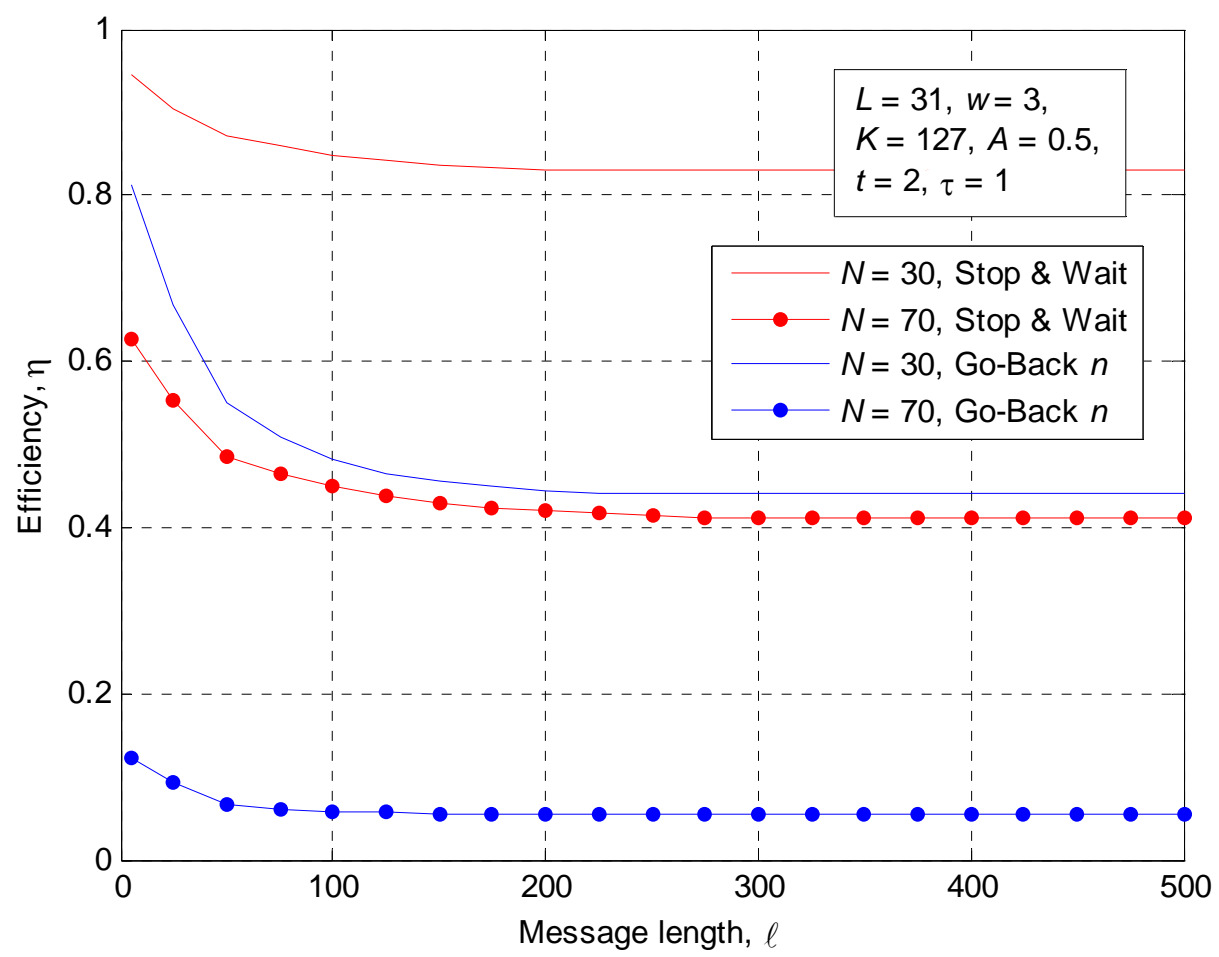

Fig. 7. Efficiency vs. message length for different number of users. 
The protocol efficiency has been plotted against the message length for both protocols in Fig. 7. General trends of the curves can be noticed. The efficiency decreases as the message length is increased till it reaches its floor. This can be argued in a similar way as in Fig. 5. Both protocols achieve higher efficiency in case of low population networks. It can be noticed that our proposed protocol provides an improvement in the protocol efficiency for both small and large population networks.

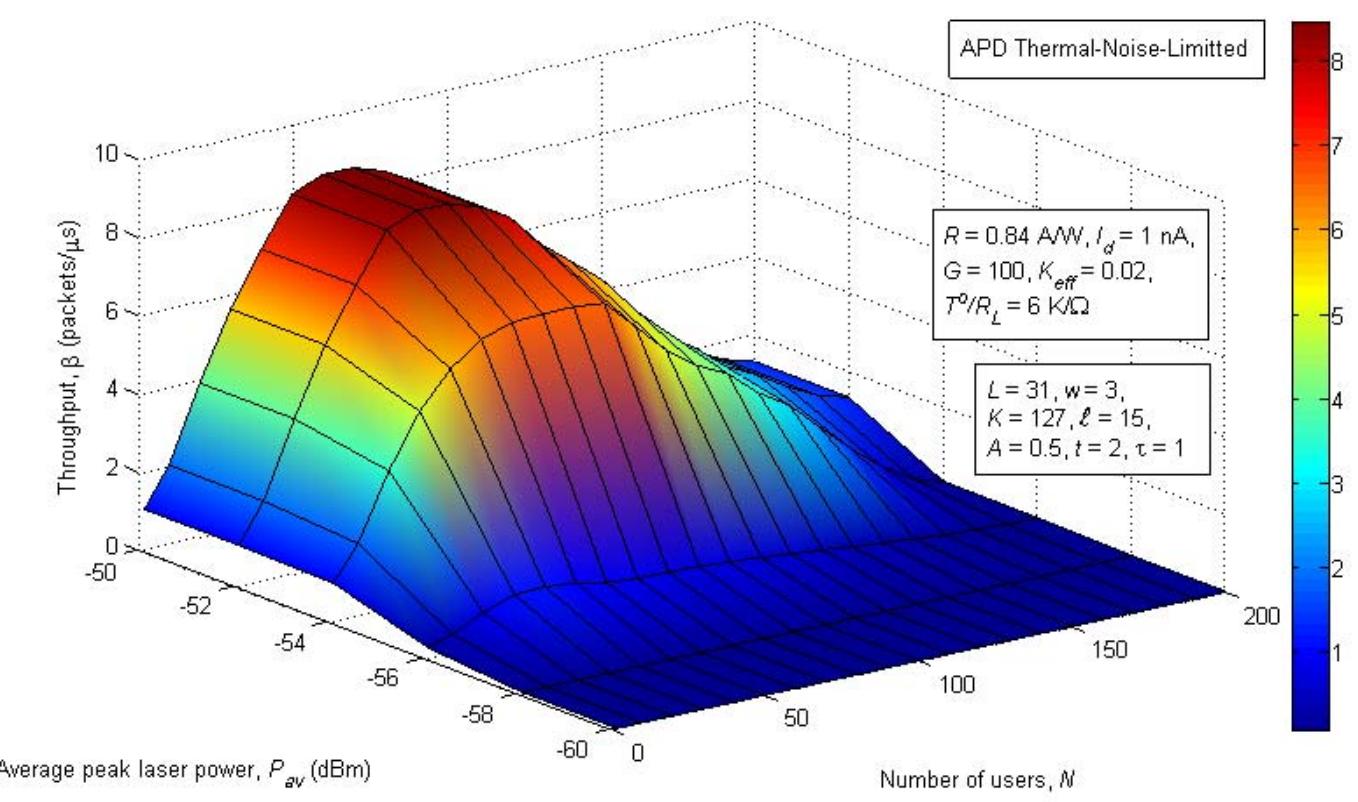

Fig. 8. Throughput vs. number of users and average peak laser power per chip.

Finally, the impact of thermal noise on our proposed protocol is taken into account in Fig. 8. It can be inferred that by increasing the average peak laser power up to $-52 \mathrm{dBm}$, the receiver can tolerate the effect of the performance degradation and achieves the same throughput as the ideal case (considering only the effect of MAI).

\section{VI- CONCLUSIONS}

In this paper we have proposed an optical random access CDMA protocol based on a stop \& wait ARQ. A mathematical description of this protocol has been presented using a detailed state diagram. Several performance measures were considered, namely, the steady state system throughput, the blocking probability, the average packet delay, and 
the protocol efficiency. In our numerical calculations, we have focused only on the effect of both MAI and receiver thermal noise. Both correlation and chip-level receivers were also examined. Finally a comparison between our proposed protocol and the $R^{3} T$ protocol [9] was discussed. The following concluding remarks can be extracted from our results.

1- The performance of the proposed CDMA protocol using correlation receivers is almost the same as that of the $R^{3} T$ protocol with chip-level receivers.

2- The proposed protocol outperforms the $R^{3} T$ protocol (in terms of the system throughput and average packet delay) in high population networks, whereas for smaller size networks the $R^{3} T$ protocol slightly outperforms the proposed one.

3- Both protocols exhibit satisfactory blocking probability only for small traffic loads. Furthermore, the average packet delay is acceptable under different network parameters.

4- For larger interstation distances, the proposed protocol accommodates a higher number of users. Also the quality of service (QOS) requirements can be achieved in a larger dynamic range (wider range of users).

5- Our proposed protocol provides an improvement in the protocol efficiency for both small and large population networks. Also it is clear that for an interstation distance $z=200 \mathrm{~m}$, the efficiency of our protocol with $N=70$ is as good as the efficiency of the $R^{3} T$ protocol with $N=30$.

6- We can tolerate the effect of thermal noise by reasonably increasing the average peak power at the transmitter to $-52 \mathrm{dBm},[12]$.

7- The complexity of the proposed protocol is significantly reduced compared to the $R^{3} T$ protocol. Also the cost is reduced when using correlation receivers.

\section{REFERENCES}

[1] F. R. Chung, J. A. Salehi, and V. K. Wei, "Optical orthogonal codes: Design, analysis, and applications," IEEE Trans. Inform. Theory, vol. 35, pp 595-604, May 1989.

[2] J. A. Salehi and C. A Brackett, "Code division multiple-access techniques in optical fiber networks. Part II: systems performances analysis," IEEE Trans. Commun, vol. COM-37, pp 834-842, Aug.1989. 
[3] H. M. H. Shalaby, "Complexities, error probabilities, and capacities of optical OOK-CDMA communication systems," IEEE Trans. Commun, vol. COM-50, pp. 2009-2015, Dec. 2002.

[4] H. M. H. Shalaby, "Chip-level detection in optical code-division multiple-access," IEEE/OSA J. Light- wave Technol, vol. LT-16, pp. 1077.1087, June 1998.

[5] M. J. Karol, "Performance of the PAC optical packet network," Journal of Lightwave Technology, vol 11. no. 8, pp. 1394-1399, Aug. 1993.

[6] C.-S. Hsu and V. O. K. Li, "Performance analysis of slotted fiber-optic codedivision multiple-access (CDMA) packet networks," IEEE Trans. Commun, vol. COM-45, pp. 819-828, July 1997.

[7] C.-S. Hsu and V. O. K. Li, "Performance analysis of unslotted fiber-optic codedivision multiple-access (CDMA) packet networks," IEEE Trans. Commun, vol. COM-45, pp. 978-987, Aug. 1997.

[8] H. M. H. Shalaby, "Optical CDMA random access protocols with and without pretransmission coordination," IEEE/OSA J. Lightwave Technol., vol. LT-21, pp. 2455-2462, Nov. 2003.

[9] H. M. H. Shalaby, "Performance analysis of an optical CDMA random access protocol," IEEE/OSA J. Lightwave Technol., vol. LT-22, pp. 1233-1241, May 2004.

[10] M. A. A. Mohamed, H. M. H. Shalaby, and E. A. El-Badawy, "Optical CDMA protocol with selective retransmission," in Proc. Ninth IEEE Symp. On Computers sand Communications (ISCC 2004), Alexandria, Egypt, June 29-July 1, 2004, pp. 621-626.

[11] J.-R. Sack and J. Urrutia, Handbook of Computational Geometry. North Holland: Elsevier Science, 2000.

[12] Z. A. El-Sahn, Y. M. Abdel-Malek, H. M. H. Shalaby and El-S. A. El-Badawy, "Performance limitations in the $R^{3} T$ optical random access CDMA protocol," Submitted to OSA.

[13] Xi Zhang, Kang G. Shin, "Markov-chain modeling for multicast signaling delay analysis", IEEE/ACM Transactions on Networking, vol. 12, no. 4, Aug 2004 pp. 667-680 